OPEN ACCESS

Edited by:

Marialuisa Gandolfi,

University of Verona, Italy

Reviewed by:

Ota Gál,

Charles University, Czechia

Roberto Rodríguez-Labrada,

Cuban Neuroscience Center, Cuba

${ }^{*}$ Correspondence:

Jia Han

Jia.Han@Canberra.edu.au

tThese authors have contributed equally to this work and share first

authorship

Specialty section:

This article was submitted to

Neurorehabilitation,

a section of the journal

Frontiers in Neurology

Received: 18 August 2021 Accepted: 22 October 2021 Published: 23 November 2021

Citation:

Tao P, Shao X, Zhuang J, Wang Z,

Dong $Y$, Shen $X$, Guo $Y$, Shu $X$,

Wang H, XU Y, Li Z, Adams R and Han J (2021) Translation, Cultural Adaptation, and Reliability and Validity

Testing of a Chinese Version of the

Freezing of Gait Questionnaire

(FOGQ-CH).

Front. Neurol. 12:760398.

doi: 10.3389/fneur.2021.760398

\section{Translation, Cultural Adaptation, and Reliability and Validity Testing of a Chinese Version of the Freezing of Gait Questionnaire (FOGQ-CH)}

\author{
Ping Tao ${ }^{1,2+}$, Xuerong Shao ${ }^{1+}$, Jie Zhuang ${ }^{1}$, Zhen Wang ${ }^{3}$, Yuchen Dong ${ }^{2}$, Xia Shen ${ }^{4,5}$, \\ Yunjie Guo ${ }^{6}$, Xiaoyi Shu ${ }^{1}$, Hong Wang ${ }^{7}$, Yuanhong $X^{8}{ }^{8}$, Zhenlan $\mathrm{Li}^{1,9}$, Roger Adams ${ }^{10}$ and \\ Jia Han ${ }^{1,10,11 *}$ \\ ${ }^{1}$ School of Kinesiology, Shanghai University of Sport, Shanghai, China, ${ }^{2}$ School of Medicine, Jinhua Polytechnic, Jinhua, \\ China, ${ }^{3}$ School of Martial Arts, Shanghai University of Sport, Shanghai, China, ${ }^{4}$ School of Medicine, Tongji University, \\ Shanghai, China, ${ }^{5}$ Shanghai YangZhi Rehabilitation Hospital (Shanghai Sunshine Rehabilitation Center), Tongji University \\ School of Medicine, Shanghai, China, ${ }^{6}$ Department of Rehabilitation Medicine, Shenzhen Samii International Medical Center \\ (The Fourth People's Hospital of Shenzhen), Shenzhen, China, ${ }^{7}$ College of Rehabilitation Science, Shanghai University of \\ Medicine and Health Sciences, Shanghai, China, ${ }^{8}$ Rehabilitation Department, Affiliated Taihe Hospital of Hubei University of \\ Medicine, Shiyan, China, ${ }^{9}$ Department of Rehabilitation Sciences, Ningbo College of Health Sciences, Ningbo, China, \\ ${ }^{10}$ Research Institute for Sports and Exercise, University of Canberra, Canberra, ACT, Australia, " Faculty of Health, Arts and \\ Design, Swinburne University of Technology, Hawthorn, VIC, Australia
}

Freezing of gait is a disabling symptom with a complex episodic nature that is frequently experienced by people with Parkinson's disease (PD). Although China has the largest population with $\mathrm{PD}$ in the world, no Chinese version of the freezing of gait questionnaire (FOGQ), the instrument that has been most widely used to assess FOG, has yet been developed. This study aimed to translate and adapt the original version of FOGQ to create a Chinese version, the FOGQ-CH, then assess its reliability, calculate the Minimal Detectable Change (MDC) and investigate its validity. The forward-backwards translation model was adopted, and cultural adaptation included expert review and pretesting. For the reliability study, 31 Chinese native speaking patients with PD were assessed two times in a 7-10 days interval. Internal consistency and test-retest reliability of the FOGQ-CH were measured by Cronbach's alpha $(\mathrm{C} \alpha)$ and the Intraclass Correlation Coefficient (ICC). For the validity study, 34 native speakers of Chinese with PD were included. To explore the convergent validity, relationships between the FOGQ-CH and the Unified Parkinson's Disease Rating Scale Part II (UPDRS II) and Part III (UPDRS III), Timed Up and Go Test (TUGT), Timed Up and Go Test in cognitive task (TUGT-Cog), walking speed (10 MWT speed), and step length (10 MWT step length) in a 10-m Walk Test were tested. To explore predictive validity, the number of falls followed up for 6 months were assessed. The area under the ROC curve (AUC) was employed to test the capacity of FOGQ-CH to discriminate those with falls. From the reliability study, $\mathrm{C} \alpha=0.823, \mathrm{ICC}=0.786$. The $\mathrm{MDC}_{0.90}=4.538$. From the validity study, the FOGQ-CH showed moderate correlations with UPDRS II (rho $=0.560, p=0.001)$, UPDRS III (rho $=0.451, p=0.007)$, TUGT (rho $=0.556$, $p=0.007$ ), TUGT-Cog (rho $=0.557, p=0.001)$, 10MWT-speed (rho $=-0.478$, $p=0.004$ ), 10MWT-step length (rho $=-0.419, p=0.014$ ), and the number of 
falls followed up for 6 months ( $r$ o $=0.356, p=0.045)$. The AUC $=0.777(p=0.036$ ) for predicting whether the participants will have multiple falls (two or more) in the following 6 months. The FOGQ-CH showed good reliability and validity for assessing Chinese native speaking patients with PD. In addition, the FOGQ-CH showed good efficacy for predicting multiple falls in the following 6 months.

Keywords: freezing of gait, freezing of gait questionnaire, Parkinson's disease, fall, translation, reliability study, validity study

\section{INTRODUCTION}

Freezing of gait is one of the most common disabling symptoms in Parkinson's disease (PD), and it affects $\sim 63 \%$ of patients with idiopathic PD (1). Freezing of gait (FOG) has been defined as the experience of sudden and, usually, brief episodes with the inability to make effective steps (2). During the FOG episode, patients report the feeling that their feet are glued to the floor (2). FOG can lead to falling, low quality of life, and dependence (3). In addition, FOG has been hard to assess objectively (4).

The Freezing of gait questionnaire (FOGQ) is a patientreported outcome measure, which was developed by Giladi et al. (5). It has six questions and can easily be administered in clinics. To date, the FOGQ has been translated to different languages, including Swedish, Italian, Turkish, Spanish, Brazilian, German, and Czech (4, 6-11), and these versions have shown good validity and reliability. China has the largest population with PD globally, and it is estimated that the number of patients with PD in China will reach half of all patients with PD in the world by 2030 (12). However, there is, at present, no Chinese version of the FOGQ. Accordingly, one of the aims of the present study was to develop a Chinese translation version of the FOGQ and assess its reliability and validity. In addition, while the Minimal Detectable Change (MDC) of the FOGQ is important when interpreting the change of the scores following treatment, it has not been reported in previous studies.

Falling is a sign of poor balance that can bring serious physical damage to patients with PD (i.e., fractures) (13). The early presence of falling is a risk factor in patients with PD (1). FOG was one factor of falling $(3,14)$; however, it is undetermined if the Chinese version of FOGQ can be used for fall prediction in Chinese patients with PD.

We hypothesize that the test-retest reliability of the Chinese version of the FOGQ (FOGQ-CH) will be reliable enough to be on par with the Unified Parkinson's Disease Rating Scale (UPDRS) Part II and Part III involving activities of daily living (ADL) and motor performance $(15,16)$, three mobility measurements include the Timed Up and Go Test (TUGT), the Cognitive dual-task Timed Up and Go Test (TUGT-Cog) (17), and the 10-m Walk Test (10MWT) (18). In addition, it is hypothesized that the FOGQ-CH will show good predictive validity in terms of the number of falls in Chinese patients with PD.

\section{MATERIALS AND METHODS}

The study was approved by the Committee for Ethics in Human Research at Shanghai University of Sport (Approval number: 102772021RT038). All patients signed informed consent before the data collection.

\section{Part 1: Translation and Cross-Cultural Adaptation}

The (FOGQ-CH) was translated by researchers based on a standard procedure of forward-backwards translation (19). The procedure is shown in Figure 1.

\section{Forward Translation and Synthesis}

Two native Chinese translators independently translated the English version to Chinese after obtaining permission from the corresponding author of the original English FOGQ. One translator was adept on research on Parkinson's disease, and another translator was a physiotherapist with a master's degree in Hongkong. Both were native Chinese speakers who had good English language skills. After two translators finished the forward translation, we got the forward translated version 1 and forward translated version 2. Any ambiguities and discrepancies in the two versions were discussed by our research team (which included a health science professor, a neural physiotherapist, and a doctor of Parkinson's disease). The research team then made a contrastive analysis and formed a forward translated synthetic version.

\section{Backward Translation and Synthesis}

After forward translation and synthesis, the forward translated synthetic Chinese version was independently translated back to English by another two translators who had never read the original version of FOGQ. Both of them had more than 2 years of study and life experience in Australia. One translator was a master of physiotherapy and another translator is a professor of English linguistics. After two translators finished the backward translation, we got the backward translated version 1 and forward translated version 2 . The research team made a backward translated synthetic English version by discussion. The forward and backward translations would continue until the semantic consistency rate of the backward translated version reached $95 \%$ or more with the original version (20). 


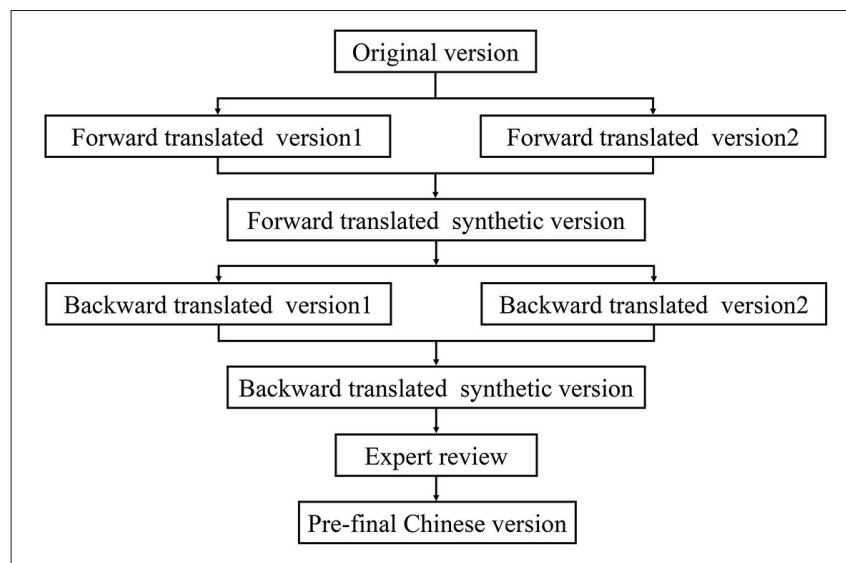

FIGURE 1 | The procedure of the translation and cross-cultural adaptation of Chinese version of the Freezing gait questionnaire (FOGQ-CH).

\section{Cultural Adaption}

There were five experts in rehabilitation science, all Chinese native speakers, who worked to evaluate the first version of FOGQ-CH. The inclusion criteria for evaluators were experts who were educated with a Master's degree level or above, had at least 10 years of work experience in clinical practice, research, or education, and had professional knowledge of $\mathrm{PD}$. According to their suggestion, some words were culturally adapted. For example, the words "gait difficulties" in question 2 were revised to "walking problem" because the term "walking problem" is easier to understand as a Chinese expression. After modification as per the suggestions of the experts, the second version (Pre- final Chinese version) was established.

We invited 10 Chinese patients with PD (their demographic characteristic was shown in Table $\mathbf{1}$ ) to complete the pre-test with the second version of FOGQ-CH so as to investigate comprehensibility and acceptability. According to their feedback, the questionnaire was revised slightly, and the final version of the FOGQ-CH was developed (see in Supplementary Material).

\section{Part 2: Reliability and Validity Study Participants}

We recruited participants from Xinhua Hospital Affiliated to Shanghai Jiaotong University School of Medicine in Shanghai City and Jinhua Hospital of traditional Chinese medicine in Zhejiang Province. Thirty-one Chinese-speaking patients with PD volunteered for the reliability study, and 34 participants for the predictive study. The demographic characteristics of the participating patients with PD are summarized in Table 1. The inclusion criteria of participants were as follows: patients diagnosed with Idiopathic PD by neurologists, lasting over 6 months; Modified Hoehn and Yahr stage $\leq 4$ in "off" phase according to the description of the patient; patients in stable treatment over 2 months since last modification; score on the Mini-Mental State Examination of over 24/30; and the patient could be able to walk at least $50 \mathrm{~m}$ with or without a walking aid. Finally, patients had symptoms of
FOG as indicated by patients and their caregivers during the last week.

\section{Material}

The first convergent validity measurement employed was the UPDRS, as it is widely used in patients with PD. It has four parts (Parts I-IV). Part II and Part III are about ADL and Motor performance assessment. Part II has 13 questions and Part III has 14 questions, where each question has five categories scored from 0 to 4 , where 0 means normal and 4 means the maximum amount of the problem.

The TUGT is a measurement tool used to evaluate the balance, posture control, and mobility of patients with PD (21). Participants are required to stand up from a chair with armrests (seat height of $50 \mathrm{~cm}$, armrest height of $65 \mathrm{~cm}$ ) (22), walk three meters as quickly and safely as possible, cross an obstacle on the floor, turn around, walk back, and sit down. The tester records the time from standing up to sitting down. For the TUGT-Cog test, the patient is asked to perform the same TUGT while counting backwards by sevens from a randomly selected number between 90 and 100 .

In 10MWT, the patient is instructed to walk $14 \mathrm{~m}$ at a fast but safe speed. The first and last $2 \mathrm{~m}$ are given to accelerate and decelerate, respectively, and the $10 \mathrm{~m}$ zone in the middle is assessed. The time and number of steps are recorded. Walking speed is calculated by dividing the time needed by $10 \mathrm{~m}$ (meters/second). The step lengths were calculated by dividing steps by $10 \mathrm{~m}$ (meters/numbers).

\section{Data Collection}

For the reliability study, the FOGQ-CH was filled in twice by 31 patients with $\mathrm{PD}$. The interval between the two tests was 7-10 days.

For the validity studies, content validity, convergent validity, and predictive validity were evaluated. Content validity was measured by five experts using the Delphi method. In the convergent validity and predictive validity studies, all assessments were conducted during the "ON" state. The numbers of falls during the 6 months post initial assessment were recorded to determine the relationship between the scores of the FOGQ-CH and the number of falls.

\section{Statistical Analysis}

Statistical analysis was performed with Microsoft Excel 2019 and SPSS version 25.0.

Reliability analysis included calculating internal consistency and test-retest reliability. Internal consistency was calculated by Cronbach's alpha $(\mathrm{C} \alpha)$ using each item of the FOGQ-CH. A strong internal consistency should show a moderate correlation when $\mathrm{C} \alpha$ values are between 0.70 and 0.95 (23).

For the test-retest reliability, an Intraclass Correlation Coefficient [ICC (3.1)] with two-way fixed model, single measure type, and absolute agreement definition was calculated for each item (24). ICC values were classified as poor $(<0.50)$, moderate (between 0.50 and 0.75 ), good (between 0.75 and 0.90 ), and 
TABLE 1 | Demographic characteristics of patients with PD, Means \pm SD (minimum-maximum).

\begin{tabular}{|c|c|c|c|}
\hline & Pre-test study & Reliability study & Validity study \\
\hline Patients (n) & 10 & 31 & 34 \\
\hline $\operatorname{Sex}(M / F)$ & $6: 4$ & $13: 18$ & $18: 16$ \\
\hline Age (y) & $\begin{array}{c}62.80 \pm 8.70 \\
(46-76)\end{array}$ & $\begin{array}{c}69.16 \pm 4.45 \\
(58-78)\end{array}$ & $\begin{array}{c}67.59 \pm 8.03 \\
(46-80)\end{array}$ \\
\hline Height (m) & $\begin{array}{l}1.64 \pm 0.07 \\
(1.55-1.75)\end{array}$ & $\begin{array}{l}1.62 \pm 0.08 \\
(1.50-1.80)\end{array}$ & $\begin{array}{l}1.63 \pm 0.07 \\
(1.52-1.80)\end{array}$ \\
\hline Weight (kg) & $\begin{array}{c}65.00 \pm 6.82 \\
(52-75)\end{array}$ & $\begin{array}{c}61.26 \pm 11.51 \\
(36-78)\end{array}$ & $\begin{array}{c}59.94 \pm 10.17 \\
(36-75)\end{array}$ \\
\hline MMSE score (point) & $\begin{array}{c}27.40 \pm 2.07 \\
(25-30)\end{array}$ & $\begin{array}{c}27.52 \pm 1.84 \\
(24-30)\end{array}$ & $\begin{array}{c}28.15 \pm 1.73 \\
(24-30)\end{array}$ \\
\hline PD duration (y) & $\begin{array}{c}6.40 \pm 6.08 \\
(0.5-19)\end{array}$ & $\begin{array}{c}7.19 \pm 4.66 \\
(2-19)\end{array}$ & $\begin{array}{c}6.99 \pm 4.86 \\
(1-20)\end{array}$ \\
\hline Modified H\&Y stage "ON" (stage) & $\begin{array}{c}1.75 \pm 0.75 \\
(1-3)\end{array}$ & $\begin{array}{c}2.07 \pm 0.73 \\
(1-3)\end{array}$ & $\begin{array}{c}1.93 \pm 0.80 \\
(1-4)\end{array}$ \\
\hline
\end{tabular}

PD, Parkinson's Disease; MMSE, Mini-Mental State Examination; H\&Y, Hoehn and Yahr; M/F, Male/Female.

excellent (over 0.90) (23). With this ICC, the MDC was calculated using the following formula $(25,26)$ :

$$
\begin{aligned}
\mathrm{MDC}_{0.90} & =\mathrm{SEM} \times 1.65 \times \sqrt{2} \\
\mathrm{SEM} & =\mathrm{S} \sqrt{1-\mathrm{ICC}} \\
\% \mathrm{MDC} & =\frac{\mathrm{MDC}_{0.90}}{\text { Maximum }} \times \%
\end{aligned}
$$

where SEM was the standard error of measurement, and $s$ was the standard deviation of the measurements taken at the first time.

Content validity was tested by the item-content validity index (I-CVI) and the scale-content validity index (S-CVI). The experts rated each item in terms of its relevance to the underlying construct using a 4-point scale where 1 meant not relevant, 2 somewhat relevant, 3 quite relevant, and 4 highly relevant. The number of experts who gave a rating of 3 or 4 divided by the total number of experts for each item is calculated for the I-CVI. The I-CVIs was then added and divided by the number of items to calculate the S-CVI where I-CVI of 0.8 was considered quite relevant, and S-CVI of 0.8 or higher was acceptable (27).

Convergent validity was measured by Spearman's correlation. The correlation coefficient ( $r h o$ ) value was classified as negligible (between 0 and 0.10), weak (between 0.10 and 0.39), moderate (between 0.40 and 0.69 ), strong (between 0.70 and 0.89 ), and very strong (between 0.90 and 1.00) correlation (28). In addition, the difference between the TUGT and TUGT-Cog was determined by a Paired-Samples $t$-test.

Predictive validity was measured by the area under the receiver operating characteristic curve (AUC) in order to evaluate the ability of the FOGQ-CH and to discriminate the number
TABLE 2 | Intraclass correlation coefficient (ICC) of freezing of gait questionnaire, Chinese language version (FOGQ-CH).

\begin{tabular}{lcc}
\hline Index & I-CVI $(\boldsymbol{n}=\mathbf{5})$ & ICC $(\mathbf{9 5} \% \mathbf{C l}, \boldsymbol{n}=\mathbf{3 1})$ \\
\hline Item 1 & 1.000 & $0.848(0.710-0.924)$ \\
Item 2 & 0.800 & $0.710(0.479-0.849)$ \\
Item 3 & 1.000 & $0.648(0.440-0.834)$ \\
Item 4 & 1.000 & $0.679(0.433-0.831)$ \\
Item 5 & 1.000 & $0.700(0.467-0.842)$ \\
Item 6 & 1.000 & $0.469(0.153-0.700)$ \\
S-CVI & 0.967 & \\
Total score & & $0.786(0.578-0.894)$ \\
\hline
\end{tabular}

ICC, Intraclass Correlation Coefficient; FOGQ-CH, Freezing of Gait Questionnaire, Chinese-language version; I-CVI, Item-Content Validity Index; S-CVI, Scale-Content Validity Index; Cl, Confidence Interval.

of falls. Two conditions were analyzed. The first was defined as positive by one or more falls, and the second condition was defined as positive by two or more falls. A cutoff score was calculated if the AUC score was significant $(p<0.05)$. The cutoff value was calculated by the maximum value of Youden's Index, which corresponds to the maximum difference between sensitivity and $1-$ specificity in the receiver operating characteristic curve (ROC).

\section{RESULTS}

\section{Reliability Study}

Reliability results showed a satisfactory internal consistency, with a $\mathrm{C} \alpha$ value of 0.823 . Test-retest reliability results are shown in Table 2. For six items, the ICC value ranged from 0.469 to 0.848 , and the total score ICC was 0.786 , indicating good test-retest reliability. The FOGQ-CH's $\mathrm{MDC}_{0.90}$ was 4.538 , and the $\% \mathrm{MDC}$ was $18.908 \%$. 
TABLE 3 | FOGQ-CH correlations with indices.

\begin{tabular}{lcc}
\hline Index & Correlation (rho) & p-value \\
\hline UPDRS II & 0.560 & 0.001 \\
UPDRS III & 0.451 & 0.007 \\
TUGT & 0.556 & 0.001 \\
TUGT-Cog & 0.557 & 0.001 \\
1OMWT-Speed & -0.478 & 0.004 \\
1OMWT-Step length & -0.419 & 0.014 \\
The number of falls & 0.346 & 0.045 \\
\hline
\end{tabular}

FOGQ, Freezing of Gait Questionnaire; UPDRS II, Unified Parkinson's Disease Rating Scale Part II; UPDRS III, Unified Parkinson's Disease Rating Scale Part III; TUGT, Timed Up and Go Test; TUGT-Cog, Timed Up and Go Test in cognitive task; 10MWT, 10-Meter Walk Test.

\section{Validity Study}

In terms of content validity, the I-CVI for each item ranged from 0.80 to 1.00 , and the S-CVI was 0.97 , as shown in Table 2 .

The convergent validity results showed that all PD-related measurements were moderately correlated with FOGQ-CH (Table 3). UPDRS II, UPDRS III, TUGT, TUGT-Cog, and the number of falls had a positive correlation with the FOGQ-CH. As expected, 10MWT speed and 10MWT step length showed a negative correlation with the FOGQ-CH. A significant difference $(t=-3.390, p=0.002)$ was found between TUGT (12.317 $\pm 5.433)$ and TUGT-Cog $(15.082 \pm 9.363)$ with the PairedSamples $t$-Test.

At the 6 months follow-up, five patients experienced 1 fall, one patient 2 falls, two patients 3 falls, one patient 5 falls, one patient 8 falls, and one patient experienced 12 falls. In summary, there are $32.4 \%(11 / 34)$ patients who experienced at least one fall and $17.6 \%(6 / 34)$ patients who experienced two or more falls. In the first condition, one or more falls was defined as positive, $\mathrm{AUC}=0.684(p=0.087)$. In the second condition, two or more falls was defined as positive, AUC $=0.777(p$ $=0.036$ ), and the cutoff score derived from the ROC analysis was 7.5 points, the sensitivity was $100 \%$, and the specificity was $60.7 \%$.

\section{DISCUSSION}

Freezing of Gait is one of the major motor disorders in patients with PD. It is one of the risk predictors of falling in patients with PD, and its pathophysiology is still unclear. Being older, falls, hallucinations, having anxiety, living in the countryside, having an akinetic-rigid style, and onset of lower limbs may be the predictors of FOG in Chinese patients $(29,30)$. The FOGQ has been found to be a reliable and valid measurement tool when used to assess FOG in other language versions (4, 5, 8-11). The questionnaire had no floor or ceiling effects, and it had a high internal consistency, good inter and intra-rater reliability, good content, and convergent validity. The Movement Disorder Society (MDS) Task Force on Rating Scales also recommended the FOGQ in evaluating FOG in 2016 (31).
Accordingly, development of a Chinese version of the FOGQ is important for Chinese patients with PD, their caregivers, and clinical workers.

\section{Reliability Study}

In the reliability study, the index of internal consistency, the $\mathrm{C} \alpha$, for the FOGQ-CH was 0.823 , a value which is similar to that found with other translated versions of the FOGQ (Spanish version: $\mathrm{C} \alpha=0.81$, German version: $\mathrm{C} \alpha=0.83$, and Brazilian version: $\mathrm{C} \alpha=0.86)(7,9,10)$. However, the original English version $(\mathrm{C} \alpha=0.94)$, the Swedish version $(\mathrm{C} \alpha=0.95)$ and the Czech version $(\mathrm{C} \alpha=0.91)$ had values for internal consistency that were very high $(4,5,11)$. Nevertheless, all translated versions met the range requirement of $0.7-0.95$, which meant that the FOGQ-CH has strong internal consistency (23).

The ICC value for each FOGQ-CH item ranged from 0.469 to 0.848 , so each item showed a moderate correlation coefficient, and the FOGQ-CH total score ICC of 0.786 was close to that of the Brazilian version (ICC $=0.78)(10)$. Hence, it can be concluded that the Chinese version of FOGQ has good testretest reliability.

The $\mathrm{MDC}_{0.90}$ of the FOGQ-CH was 4.538 . The next index, $\%$ MDC, was $18.908 \%$, which meant that $90 \%$ of patients with PD demonstrated random variation of fewer than 4.538 points in the FOGQ-CH. Therefore, if the FOGQ-CH was applied before and after an intervention, or when monitoring gait change after a certain period, a change of 4.538 points or more would be considered to be a true change. Though the $\% \mathrm{MDC}_{0.90}$ of FOGQ$\mathrm{CH}$ was high compared to some other questionnaires employed in assessments of patients with PD (Berg Balance Scale $=8.93 \%$, Activities-specific Balance Scale $=13 \%$, UPDRS II $=7.69 \%$, UPDRS III $=10.19 \%)(25)$, it was lower than the New freezing gait of questionnaire (NFOGQ, $\mathrm{MDC}_{0.95}=9.555, \% \mathrm{MDC}=$ $35.5 \%)$ according to the Hulzinga et al. (26). Compared the NFOGQ, the FOGQ showed greater capability to detect the change in FOG of patients with PD. The MDS Task Force on Rating Scales also classified the FOGQ as "recommended" and the NFOGQ as just "suggested" (31), which means that the FOGQ may still be preferable for FOG assessment. However, the Minimal Clinical Importance Difference (MCID), a meaningful index presenting the smallest beneficial or detrimental change for the patient (32), for the FOGQ is still unknown, so future research on the MCID is needed.

\section{Validity Study}

In the content validity part of the study, five Chinese experts gave a high evaluation of the content validity, showing the FOGQ-CH items to be highly relevant to FOG. However, because there was no evaluation of content validity in previous translation versions, no comparison is possible.

According to the convergent validity results, the FOGQ-CH had a moderate correlation with UPDRS II $(r h o=0.596)$ and UPDRS III ( $r h o=0.408$ ), and in this, it was similar to the original English and Swedish version $(4,20)$.

The TUGT is an important index used to predict the balance ability of patients with PD, and poor balance can lead to falling 
(33). The FOGQ-CH (rho $=0.556$ ) had a moderate relationship with the TUGT, which was different from the German version. The reason may be the testing pace used, the sample size or the selection criteria used. In this study, 34 patients with PD participated in the study, and the study with the translated German version had 27 patients. The Modified H\&Y stage was $1-4$ in the inclusion criteria in this study, but in the German translated version study, the H\&Y stage range was $2-4$ and the Modified $\mathrm{H} \& \mathrm{Y}$ stage was not specified in the inclusion criteria, so there are some differences making comparison difficult. The FOGQ is focused more on gait, while the TUGT is focused more on overall balance ability in patients with PD. Therefore, to some extent, the FOGQ could also reflect the walking balance ability of patients with PD. The TUGT-Cog ( $r h o=0.557)$ had a moderate relationship with the FOGQ- $\mathrm{CH}$. Patients need to complete motor and cognition tasks simultaneously in TUGT$\mathrm{Cog}$, and more time is needed to process inputs when doing a dual-task. A study by Dreher and Grafman (34) showed that when people performed a dual-task, they activated the prefrontalparietal neural network more than when performing a single task, so the TUGT-Cog was shown to be more sensitive at representing the defect in ability to carry out dual tasks (17). The time spent here on dual-task performance (15.082 \pm 9.363$)$ was longer than spent on the single motor task $(12.317 \pm 5.433)$. In addition, there was a significant difference $(p<0.001)$ between performance on the TUGT and TUGT-Cog, where the TUGT-Cog was more difficult for patients with PD.

In the 10MWT, the FOGQ-CH had a moderate correlation with speed ( $r h o=-0.478)$ and step length ( $r h o=-0.419)$. Gait speed and gait of patients with PD were influenced by motor impairment, plantarflexion muscle strength, and age (35). Small step length is one of the symptoms in $\mathrm{PD}$, and step length could be a predictor of executive dysfunction in PD (36), with the smaller the step length, the more serious the disease. The AUC values to test the efficacy of fast gait speed in 10MWT in predicting falls for patients with PD within 6 months, but the result was not ideal (AUC $=0.256, p=0.064$ ). It was similar to the result by Paul et al. (37) in which they also did not agree in that the fast speed of the 10MWT could predict the falls in the future.

In terms of the number of falls, we found that the FOGQ$\mathrm{CH}$ had only a weak correlation with fall numbers. There were $32.4 \%$ of patients with FOG who experienced at least one fall, and $17.6 \%$ had experienced two or more falls. According to the AUC results, the FOGQ-CH had only limited power to predict fallers and non-fallers; however, it had good predictive validity in discriminating multiple fallers at 6 months when the cut-off points of 8 was applied, which was similar to a study by Duncan and Earhart (38).

\section{CONCLUSION}

The FOGQ-CH is a reliable and valid tool when used to assess the severity of FOG in Chinese-speaking patients with PD. The FOGQ-CH had good efficacy for detecting those Chinese patients with PD who have multiple falls in the following 6 months. Future studies are needed to explore the MCID of the FOGQ in patients with $\mathrm{PD}$, and to use the FOGQ to monitor the amount of FOG change.

\section{LIMITATIONS}

Although the FOGQ-CH study and most of the FOGQ translation study did not include a control group, it would be ideal to have a control group. The aggregate validity of FOGQ$\mathrm{CH}$ was tested. However, the divergent validity has not been tested, e.g., UPDRS I. Hence, in the future, we can explore the divergent validity of FOGQ-CH. In addition, we can test the convergent validity of self-selected speed of 10MWT and FOQG-CH, and explore the predict validity of self-selected speed of 10MWT and other related indicators in predicting the fall condition of Chinese patients with PD in the future.

\section{DATA AVAILABILITY STATEMENT}

The original contributions presented in the study are included in the article/Supplementary Material, further inquiries can be directed to the corresponding author/s.

\section{ETHICS STATEMENT}

The studies involving human participants were reviewed and approved by Scientific Research Ethics Committee of Shanghai University of Sport. The patients/participants provided their written informed consent to participate in this study.

\section{AUTHOR CONTRIBUTIONS}

PT and XSha collected data, analyzed data, and drafted the manuscript. JZ, ZW, YD, and XShe helped to collect the data. YG, XShu, HW, YX, and ZL helped in the translation and cultural adaptation of the FOGQ- $\mathrm{CH}$. RA helped to edit the manuscript. $\mathrm{JH}$ participated in the study design, analyzed data, and edited the manuscript. All authors contributed to the article and approved the submitted version.

\section{FUNDING}

This study was funded by Public Welfare Foundation of Science and Technology Department of Zhejiang Province (Grant No.: LGF20H170003), Public Welfare Projects of Jinhua Science and Technology Bureau (Grant No.: 2019-4-071), National Nature Science Foundation of China (Grant No.: 31870936), Ministry of Education of China (Humanities and Social Science Project, Grant No.:18YJA890006), Program of Science and Technology Commission of Shanghai Municipality [Excellent Academic Leader (Youth) of Science and Technology Innovation Action Plan, Grant No.: 20XD1423200], and Program of Shanghai Administration of Sports (Sports Science and Technology Project, Grant No.: 21Q006). 


\section{ACKNOWLEDGMENTS}

We wish to thank Prof. Dr. Nir Giladi for his permission to translate the Freezing of Gait questionnaire (FOGQ) and the participants who contributed to our research.

\section{REFERENCES}

1. Weiss D, Schoellmann A, Fox MD, Bohnen NI, Factor SA, Nieuwboer A, et al. Freezing of gait: understanding the complexity of an enigmatic phenomenon. Brain. (2020) 143:14-30. doi: 10.1093/brain/awz314

2. Mancini M, Bloem BR, Horak FB, Lewis SJG, Nieuwboer A, Nonnekes J. Clinical and methodological challenges for assessing freezing of gait: Future perspectives. Mov Disord. (2019) 34:783-90. doi: 10.1002/mds.27709

3. Lord SR, Bindels H, Ketheeswaran M, Brodie MA, Lawrence AD, Close JCT, et al. Freezing of gait in people with Parkinson's disease: nature, occurrence, and risk factors. J Parkinsons Dis. (2020) 10:631-40. doi: 10.3233/JPD-191813

4. Nilsson MH, Hagell P. Freezing of gait questionnaire: validity and reliability of the Swedish version. Acta Neurol Scand. (2009) 120:3314. doi: 10.1111/j.1600-0404.2009.01175.x

5. Giladi N, Shabtai H, Simon ES, Biran S, Tal J, Korczyn AD. Construction of freezing of gait questionnaire for patients with Parkinsonism. Parkinsonism Relat Disord. (2000) 6:165-70. doi: 10.1016/s1353-8020(99)00062-0

6. Acaroz Candan S, Çatiker A, Ozcan TS. Psychometric properties of the Turkish version of the freezing of gait questionnaire for patients with Parkinson's disease. Neurol Sci Neurophysiol. (2019) 36:44-50. doi: 10.5152/nsn.2019.11115

7. Cervantes-Arriaga A, Rodríguez-Violante M. Validation of the spanish version of the questionnarie freezing of gait (FOG-Q) in Parkinson's disease. Arch Neurociencias. (2011) 16:173-8. Available online at: https://www.medigraphic. com/cgi-bin/new/resumenI.cgi?IDARTICULO=33409

8. Tambasco N, Simoni S, Eusebi P, Ripandelli F, Brahimi E, Sacchini E, et al. The validation of an Italian version of the Freezing of Gait Questionnaire. Neurol Sci. (2015) 36:759-64. doi: 10.1007/s10072-014-2037-5

9. Vogler A, Janssens J, Nyffeler T, Bohlhalter S, Vanbellingen T. German translation and validation of the "freezing of gait questionnaire" in patients with Parkinson's disease. Parkinson's Dis. (2015) 2015:982058. doi: 10.1155/2015/982058

10. Baggio JA, Curtarelli MB, Rodrigues GR, Tumas V. Validity of the Brazilian version of the freezing of gait questionnaire. Arq Neuropsiquiatr. (2012) 70:599-603. doi: 10.1590/s0004-282x2012000800008

11. Gal O, Polakova K, Brozova H, Bezdicek O, Hoskovcova M, Jech R, et al. Validation of the freezing of gait questionnaire in patients with Parkinson's disease treated with deep brain stimulation. Neurol Sci. (2020) 41:11338. doi: 10.1007/s10072-019-04209-3

12. Li G, Ma J, Cui S, He Y, Xiao Q, Liu J, et al. Parkinson's disease in China: a forty-year growing track of bedside work. Transl Neurodegener. (2019) 8:22. doi: 10.1186/s40035-019-0162-z

13. Beydoun HA, Beydoun MA, Mishra NK, Rostant OS, Zonderman AB, Eid SM. Comorbid Parkinson's disease, falls and fractures in the 2010 national emergency department sample. Parkinsonism Related Disord. (2017) 35:305. doi: 10.1016/j.parkreldis.2016.11.005

14. Okuma Y, Silva de Lima AL, Fukae J, Bloem BR, Snijders AH. A prospective study of falls in relation to freezing of gait and response fluctuations in Parkinson's disease. Parkinsonism Related Disord. (2018) 46:30-5. doi: 10.1016/j.parkreldis.2017.10.013

15. Ramsay N, Macleod AD, Alves G, Camacho M, Forsgren L, Lawson RA, et al. Validation of a UPDRS-/MDS-UPDRS-based definition of functional dependency for Parkinson's disease. Parkinsonism Related Disord. (2020) 76:49-53. doi: 10.1016/j.parkreldis.2020.05.034

16. Sanchez-Ferro A, Matarazzo M, Martinez-Martin P, Martinez-Avila JC, Gomez de la Camara A, Giancardo L, et al. Minimal clinically important difference for UPDRS-III in daily practice. Mov Disord Clin Pract. (2018) 5:448-50. doi: $10.1002 / \mathrm{mdc} 3.12632$

\section{SUPPLEMENTARY MATERIAL}

The Supplementary Material for this article can be found online at: https://www.frontiersin.org/articles/10.3389/fneur. 2021.760398/full\#supplementary-material

17. Zirek E, Ersoz Huseyinsinoglu B, Tufekcioglu Z, Bilgic B, Hanagasi H. Which cognitive dual-task walking causes most interference on the Timed Up and Go test in Parkinson's disease: a controlled study. Neurol Sci. (2018) 39:21517. doi: 10.1007/s10072-018-3564-2

18. Lang JT, Kassan TO, Devaney LL, Colon-Semenza C, Joseph MF. Testretest reliability and minimal detectable change for the 10-meter walk test in older adults with Parkinson's disease. J Geriatr Phys Ther. (2016) 39:16570. doi: 10.1519/JPT.0000000000000068

19. Sousa VD, Rojjanasrirat W. Translation, adaptation and validation of instruments or scales for use in cross-cultural health care research: a clear and user-friendly guideline. J Eval Clin Pract. (2011) 17:26874. doi: 10.1111/j.1365-2753.2010.01434.x

20. Giladi N, Tal J, Azulay T, Rascol O, Brooks DJ, Melamed E, et al. Validation of the freezing of gait questionnaire in patients with Parkinson's disease. Mov Disord. (2009) 24:655-61. doi: 10.1002/mds.21745

21. Opara J, Malecki A, Malecka E, Socha T. Motor assessment in Parkinson's disease. Ann Agric Environ Med. (2017) 24:4115. doi: 10.5604/12321966.1232774

22. Morris S, Morris ME, Iansek R. Reliability of measurements obtained with the Timed "Up \& Go" test in people with Parkinson disease. Phys Ther. (2001) 81:810-8. doi: 10.1093/ptj/81.2.810

23. Tavakol M, Dennick R. Making sense of Cronbach's alpha. Int J Med Educ. (2011) 2:53-5. doi: 10.5116/ijme.4dfb.8dfd

24. Koo TK, Li MY. A guideline of selecting and reporting intraclass correlation coefficients for reliability research. J Chiropr Med. (2016) 15:15563. doi: 10.1016/j.jcm.2016.02.012

25. Steffen T, Seney M. Test-retest reliability and minimal detectable change on balance and ambulation tests, the 36-item short-form health survey, and the unified Parkinson disease rating scale in people with parkinsonism. Phys Ther. (2008) 88:733-46. doi: 10.2522/ptj.20070214

26. Hulzinga F, Nieuwboer A, Dijkstra BW, Mancini M, Strouwen C, Bloem BR, et al. The new freezing of gait questionnaire: unsuitable as an outcome in clinical trials? Mov Disord Clin Pract. (2020) 7:199205. doi: $10.1002 / \mathrm{mdc} 3.12893$

27. Polit DF, Beck CT. The content validity index: are you sure you know what's being reported? Critique and recommendations. Res Nurs Health. (2006) 29:489-97. doi: 10.1002/nur.20147

28. Schober P, Boer C, Schwarte LA. Correlation coefficients: appropriate use and interpretation. Anesth Analg. (2018) 126:17638. doi: 10.1213/ANE.0000000000002864

29. Zhang H, Yin X, Ouyang Z, Chen J, Zhou S, Zhang C, et al. A prospective study of freezing of gait with earlyParkinson disease in Chinese patients. Medicine. (2016) 95:e4056. doi: 10.1097/MD.0000000000 004056

30. Ou R, Wei Q, Cao B, Song W, Hou Y, Liu H, et al. Predictors of freezing of gait in Chinese patients with Parkinson's disease. Brain Behav. (2018) 8:e00931. doi: 10.1002/brb3.931

31. Bloem BR, Marinus J, Almeida Q, Dibble L, Nieuwboer A, Post B, et al. Measurement instruments to assess posture, gait, and balance in Parkinson's disease: Critique and recommendations. Mov Disord. (2016) 31:134255. doi: $10.1002 / \mathrm{mds} .26572$

32. Dekker J. The minimal clinically important difference re-considered. Osteoarthritis Cartilage. (2019) 27:1403-4. doi: 10.1016/j.joca.2019.05.014

33. Nocera JR, Stegemoller EL, Malaty IA, Okun MS, Marsiske M, Hass CJ, et al. Using the Timed Up \& Go test in a clinical setting to predict falling in Parkinson's disease. Arch Phys Med Rehabil. (2013) 94:1300-5. doi: 10.1016/j.apmr.2013. 02.020 
34. Dreher JC, Grafman J. Dissociating the roles of the rostral anterior cingulate and the lateral prefrontal cortices in performing two tasks simultaneously or successively. Cereb Cortex. (2003) 13:329-39. doi: 10.1093/cercor/13. 4.329

35. Shearin S, Medley A, Trudelle-Jackson E, Swank C, Querry R. Differences in predictors for gait speed and gait endurance in Parkinson's disease. Gait Posture. (2021) 87:49-53. doi: 10.1016/j.gaitpost.2021. 04.019

36. Amboni M, Iuppariello $\mathrm{L}$, Iavarone A, Fasano A, Palladino R, Rucco R, et al. Step length predicts executive dysfunction in Parkinson's disease: a 3-year prospective study. J Neurol. (2018) 265:2211-20. doi: 10.1007/s00415-018-8973-x

37. Paul SS, Canning CG, Sherrington C, Lord SR, Close JC, Fung VS. Three simple clinical tests to accurately predict falls in people with Parkinson's disease. Mov Disord. (2013) 28:655-62. doi: 10.1002/mds.25404

38. Duncan RP, Earhart GM. Should one measure balance or gait to best predict falls among people with Parkinson disease? Parkinsons Dis. (2012) 2012:923493. doi: 10.1155/2012/923493
Conflict of Interest: The authors declare that the research was conducted in the absence of any commercial or financial relationships that could be construed as a potential conflict of interest.

Publisher's Note: All claims expressed in this article are solely those of the authors and do not necessarily represent those of their affiliated organizations, or those of the publisher, the editors and the reviewers. Any product that may be evaluated in this article, or claim that may be made by its manufacturer, is not guaranteed or endorsed by the publisher.

Copyright (C) 2021 Tao, Shao, Zhuang, Wang, Dong, Shen, Guo, Shu, Wang, Xu, Li, Adams and Han. This is an open-access article distributed under the terms of the Creative Commons Attribution License (CC BY). The use, distribution or reproduction in other forums is permitted, provided the original author(s) and the copyright owner(s) are credited and that the original publication in this journal is cited, in accordance with accepted academic practice. No use, distribution or reproduction is permitted which does not comply with these terms. 